\title{
ON AN EQUALITY INVOLVING GROUP CHARACTERS
}

\section{J. J. PRICE}

1. Introduction. This paper concerns an analogue of the following classical problem. Let $\mu$ be a measure on the interval $[a, b]$. Define $\Pi_{n}$ to be the class of polynomials $f(x)$ of degree $n$ or less such that

(a) $f(x) \geqq 0, x \in[a, b]$,

(b) $\int_{a}^{b} f(x) d \mu(x)=1$.

The problem is to find, for each $x \in[a, b], M_{n}(x)=\sup f(x)$ where $f$ runs over $\Pi_{n}$. The solution is given in Szegö [7, p. 181]. ${ }^{1}$ For our purposes it is enough to state that the solution is of the form

$$
M_{n}(x)= \begin{cases}\max \left\{F_{k}(x), G_{k}(x)\right\} & \text { if } n=2 k, \\ \max \left\{H_{k}(x), J_{k}(x)\right\} & \text { if } n=2 k+1,\end{cases}
$$

where all functions named are non-negative,

$$
\int_{a}^{b} F_{k}(x) d \mu(x)=k
$$

and

$$
\int_{a}^{b} G_{k}(x) d \mu(x)=\int_{a}^{b} H_{k}(x) d \mu(x)=\int_{a}^{b} J_{k}(x) d \mu(x)=k+1 .
$$

It follows that

$$
\int_{a}^{b} M_{n}(x) d \mu(x) \leqq n+1
$$

and that

$$
\int_{a}^{b} M_{n}(x) d \mu(x) \geqq \begin{cases}\frac{1}{2}(n+2) & \text { if } n=2 k, \\ \frac{1}{2}(n+1) & \text { if } n=2 k+1 .\end{cases}
$$

Since any polynomial is a linear combination of orthogonal polynomials, the problem may be generalized by replacing polynomials on an interval with linear combinations of a given orthogonal set on a measure space. The solution seems to require rather detailed knowl-

Presented to the Society, April 19, 1963; received by the editors June 6, 1962 and, in revised form, August 30, 1962.

${ }^{1}$ Interesting refinements and extensions are found in Schoenberg-Szegö [5] and Brickman [1]. 
edge of the orthogonal set in question. There is a class of orthogonal systems, however, the characters of second countable compact abelian groups, which have sufficiently nice properties that general results can be found. For these systems, the analogue of (1) will be proved and conditions under which equality holds will be given. It will also be shown that (2) fails in general.

2. Definitions. $X$ will denote a second countable compact abelian group, $\mu$ its Haar measure, and $\Psi=\left\{\psi_{j}\right\}_{j=0}^{\infty}$ its character group, ${ }^{2}$ with $\psi_{0}(x) \equiv 1$.

$\Pi_{n}$ will be the class of all functions $f$ defined on $X$ with the following three properties.

(a) $f(x)=\sum_{j=0}^{n} a_{j} \psi_{j}(x), a_{j}$ complex.

(b) $f(x) \geqq 0, x \in X$.

(c) $\int_{X} f(x) d \mu(x)=1$.

Define $M_{n}(x)=\sup f(x)$ for $f \in \Pi_{n}$.

3. Properties of $M_{n}(x)$. First of all, $M_{n}(x)=M_{n}$, independent of $x$. This is an immediate consequence of the invariance of $\Pi_{n}$ under translation, i.e. if $f_{y}(x)=f(x+y)$, then $f \in \Pi_{n}$ if and only if $f_{v} \in \Pi_{n}$ for every $y \in X$. Since $f\left(x_{1}\right)=f_{x_{1}-x_{2}}\left(x_{2}\right), M_{n}\left(x_{1}\right)=M_{n}\left(x_{2}\right)$.

Next, it is possible to compute $M_{n}$ as a supremum over a smaller class of functions. Let $\Pi_{n}^{\prime}(x)$ be the subset of $\Pi_{n}$ consisting of those functions which take their maximum value at $x$. Define $M_{n}^{\prime}(x)$ $=\sup f(x)$ where $f$ runs over $\Pi_{n}^{\prime}(x)$. Just as for $M_{n}(x), M_{n}^{\prime}(x)=M_{n}^{\prime}$ independent of $x$.

We assert that $M_{n}^{\prime}=M_{n}$. Obviously $M_{n}^{\prime} \leqq M_{n}$. The reverse inequality can be seen as follows. Given $\epsilon>0$, there exists $f \in \Pi_{n}$ and a point $x_{1} \in X$ such that $f\left(x_{1}\right)>M_{n}-\epsilon$. If $f$ assumes its maximum value at $x_{2}, f_{x_{2}-x_{1}}\left(x_{1}\right) \geqq f\left(x_{1}\right)>M_{n}-\epsilon$. Taking supremum over $f \in \Pi_{n}^{\prime}\left(x_{1}\right)$, $M_{n}^{\prime}\left(x_{1}\right)>M_{n}-\epsilon$ for every $\epsilon>0$. Hence, $M_{n}^{\prime}=M_{n}^{\prime}\left(x_{1}\right) \geqq M_{n}$.

Now we establish the analogue of (1) which, since $M_{n}(x)$ is constant, reduces to $M_{n} \leqq n+1$. It will be somewhat more convenient to write this inequality as $M_{n-1} \leqq n$.

Let $x_{0}$ be any point in $X$. If $f \in \Pi_{n-1}^{\prime}\left(x_{0}\right)$,

$$
f^{2}\left(x_{0}\right)=\left(\sum_{j=0}^{n-1} a_{j} \psi_{j}\left(x_{0}\right)\right)^{2} \leqq\left(\sum_{j=0}^{n-1}\left|a_{j}\right|^{2}\right)\left(\sum_{j=0}^{n-1}\left|\psi_{j}\left(x_{0}\right)\right|^{2}\right) .
$$

Since for any character $\psi,|\psi(x)| \equiv 1$,

$$
f^{2}\left(x_{0}\right) \leqq n \int_{X} f^{2}(x) d \mu(x) \leqq n(\max f(x)) \int_{X} f(x) d \mu(x)=n f\left(x_{0}\right) .
$$

$2 \Psi$ is second countable and discrete, hence countable. See [3], Chapter V. 
Therefore $f\left(x_{0}\right) \leqq n$, and taking the supremum over $f \in \Pi_{n-1}^{\prime}\left(x_{0}\right)$,

$$
M_{n-1} \leqq n .
$$

We ask now whether equality can hold in (4). A necessary condition is equality in (3). This will be the case only if there exists a constant $\lambda$ independent of $j$ such that $a_{j}=\lambda\left[\psi_{j}\left(x_{0}\right)\right]$-. If there is such a constant, then

$$
f(x)=\sum_{j=0}^{n-1} a_{j} \psi_{j}(x)=\lambda \sum_{j=0}^{n-1}\left[\psi_{j}\left(x_{0}\right)\right]^{-} \psi_{j}(x)=\lambda D_{n}\left(x_{0}, x\right)
$$

where $D_{n}(y, x)$ is the Dirichlet kernel associated with the orthonormal set $\left\{\psi_{j}\right\}_{j=0}^{\infty}$. Actually $\lambda=1$ since

$$
1=\int_{X} f(x) d \mu(x)=\lambda \int_{X} D_{n}\left(x_{0}, x\right) d \mu(x)=\lambda .
$$

Therefore $f(x)=D_{n}\left(x_{0}, x\right)$. Since $f(x) \geqq 0$ and $x_{0}$ was arbitrary, a necessary condition for equality in (4) is that $D_{n}(x, y) \geqq 0$ for all pairs $(x, y)$.

Here we quote a theorem of the author [4]. Let $\left\{\psi_{j}\right\}_{j=0}^{\infty}$ be an orthonormal set in $L^{2}(X, \mu), \psi_{0}(x) \equiv 1$, and $\mu(X)=1$. Suppose $D_{n}(x, y)$ $\geqq 0$ for $n=n_{1}<n_{2}<n_{3}<\cdots$. Then there exists a sequence $\left\{P_{k}\right\}_{k=1}^{\infty}$ of partitions of $X$ with the following properties. $P_{k}$ divides $X$ into $n_{k}$ subsets of positive measure. $P_{k+1}$ is a refinement of $P_{k}$. The functions $\left\{\psi_{j}\right\}_{j=0}^{n_{k}-1}$ are step functions relative to $P_{k}$, i.e. they are constant on each of the subsets of $X$ induced by $P_{k}$. (For brevity, $S\left(P_{k}\right)$ will denote the space of complex-valued step functions relative to $P_{k}$.)

Assume now that equality holds in (4) for a sequence of indices $1=n_{1}<n_{2}<n_{3}<\cdots$. Let $\Psi_{k}=\left\{\psi_{j}\right\}_{j=0}^{n_{k}-1}$. Then $\Psi_{k}$ must be a subgroup of the character group $\Psi$. For $D_{n_{k}}(x, y) \geqq 0$, so by the above theorem there exists a partition $P_{k}$ such that $\Psi_{k} \subset S\left(P_{k}\right)$ and the dimension of $S\left(P_{k}\right)$ is $n_{k}$. Since $\Psi_{k}$ has $n_{k}$ elements, $\Psi_{k}$ is an orthonormal basis of $S\left(P_{k}\right)$. Suppose $\psi_{j} \notin \Psi_{k}$. Then $\psi_{j}$ is orthogonal to $\Psi_{k}$ which shows that $\psi_{j} \in S\left(P_{k}\right)$. Thus $\Psi_{k}=\Psi \cap S\left(P_{k}\right)$. Products and inverses of elements of $\Psi_{k}$ are obviously in $S\left(P_{k}\right)$, hence in $\Psi_{k}$.

Therefore, our assumptions imply the existence of a sequence of subgroups $\Psi_{1} \subset \Psi_{2} \subset \Psi_{3} \subset \cdots, \Psi_{k}$ of order $n_{k}$, such that $\Psi=\bigcup_{k=1}^{\infty} \Psi_{k}$. Hence, $\Psi$ is a torsion group and consequently $X$ must be totally disconnected (Pontrjagin [3, Chapter V]).

Conversely, suppose $\Psi$ is the union of an expanding sequence of finite subgroups $\left\{\Psi_{k}\right\}_{k=1}^{\infty}$ where the order of $\Psi_{k}$ is $n_{k}$. Then $\Psi_{k}$ can be identified with the character group of the finite group $X / X_{k}$ where 
$X_{k}$ is the annihilator of $\Psi_{k}$. By the usual character relations for a finite group,

$$
D_{n_{k}}\left(x_{0}, x\right)=\sum_{j=0}^{n_{k}-1}\left[\psi_{j}\left(x_{0}\right)\right]^{-} \psi_{j}(x)= \begin{cases}n_{k} & \text { if } x=x_{0}\left(\bmod X_{k}\right), \\ 0 & \text { otherwise. }\end{cases}
$$

Since $D_{n_{k}}\left(x_{0}, x\right) \in \Pi_{n_{k}-1}$ it follows that $M_{n_{k}-1} \geqq n_{k}$. Hence, by (4), $M_{n_{k-1}}=n_{k}$.

We summarize these results in the following theorem.

THEOREM. Let $X$ be a second countable compact abelian group and $\Psi=\left\{\psi_{j}\right\}_{j=0}^{\infty}$ its character group, $\psi_{0}(x) \equiv 1$. Then for $n \geqq 1, M_{n-1} \leqq n$. Equality holds for a sequence of indices $1=n_{1}<n_{2}<n_{3}<\cdots$ if and only if there exists a sequence of subgroups $\Psi_{1} \subset \Psi_{2} \subset \Psi_{3} \ldots$ where $\Psi_{k}$ is of order $n_{k}$, such that $\Psi=\bigcup_{k=1}^{\infty} \Psi_{k}$. Thus $n_{k}$ must divide $n_{k+1}$ and $X$ must be totally disconnected.

The preceding theorem asserts the existence of a positive constant $p$, independent of the group and the method of enumeration of its characters, such that $M_{n-1} \leqq p n$ (namely, $p=1$ ). One may ask whether, similarly, there exists a positive constant $q$ such that

$$
M_{n-1} \geqq q n \text {. }
$$

This would provide an analogue for (2).

We shall show first that there are groups for which certain orderings of the characters admit such a $q$ while other orderings do not. An example is the circle group. Let $1 \equiv \psi_{0}(x), \psi_{1}(x), \psi_{2}(x), \ldots$ be an enumeration of its characters and define $S_{n}=\left\{\psi_{j}(x)\right\}_{j=0}^{n}$. The usual ordering is the symmetric one in which $S_{2 n}=\left\{e^{i r x}\right\}_{r=-n}^{n}, S_{2 n+1}$ $=\left\{e^{i r x}\right\}_{r=-n}^{n+1}$. In this case it follows from (6), which is proved below, that $M_{2 n}=M_{2 n+1}=n+1$. Therefore, (5) is satisfied with $q=\frac{1}{2}$.

On the other hand, an asymmetric ordering may not admit any such $q$. For, to construct real-valued functions, it is necessary to have exponentials together with their conjugates. Each character $e^{i r x} \in S_{n}$ for which $e^{-i r x} \notin S_{n}$ is "wasted" in this respect. Consider, for example, the enumeration given by $S_{n}=\left\{e^{i r x}\right\}_{r=-\nu_{n}}^{n-\nu_{n}}$ where $\left\{\nu_{n}\right\}$ is a nondecreasing sequence of positive integers tending to infinity, $\nu_{n}<\frac{1}{2} n$. Then by the result quoted above, $M_{n}=\nu_{n}+1$. If $\nu_{n}=o(n)$, there is no constant $q$ satisfying (5).

Now let $X_{k}$ be the $k$-dimensional torus, $k \geqq 1$. Number the characters by expanding cubes, i.e. so that

$$
S_{(2 n+1)^{k}-1}=\left\{e^{i\left(\nu_{1} \theta_{1}+\cdots+\nu_{k} \theta_{k}\right)} \mid-n \leqq \nu_{j} \leqq n ; j=1,2, \cdots, k\right\} .
$$

We shall show that even if the character groups of the tori are 
enumerated in this standard way, there is no positive constant $q$ satisfying (5) simultaneously for all the tori. To do this, it will be enough to prove that

$$
M_{(2 n+1)^{k}-1}=(n+1)^{k} .
$$

Once (6) is established it follows that

$$
M_{(2 n+1)^{k}-1} \sim \frac{1}{2^{k}}(2 n+1)^{k}, \quad n \rightarrow \infty .
$$

Consequently, (5) cannot hold since the positive constant $q$ would have to satisfy $q \leqq 2^{-k}$ for $k=1,2, \cdots$.

We are indebted to Dr. Louis Brickman for pointing out that (6) was obtained by Fejér for $k=1$. Fejér's method extends immediately to higher dimensions. We present the extension here because of its brevity and simplicity. It is based on the following mechanical quadrature formula. If

$$
f\left(\theta_{1}, \cdots, \theta_{k}\right)=\sum_{\nu_{1}, \cdots, \nu_{k}=-n}^{n} a_{\nu_{1}}, \cdots, \nu_{k} e^{i\left(\nu_{1} \theta_{1}+\cdots+\nu_{k} \theta_{k}\right)}
$$

then

$$
\text { (8) } \begin{aligned}
\frac{1}{(2 \pi)^{k}} \int_{0}^{2 \pi} \cdots \int_{0}^{2 \pi} f\left(\theta_{1}, \cdots, \theta_{k}\right) d \theta_{1} \cdots d \theta_{k} \\
=\frac{1}{(n+1)^{k}} \sum_{\mu_{1}, \cdots, \mu_{k}=0}^{n} f\left(\frac{2 \pi \mu_{1}}{n+1}, \cdots, \frac{2 \pi \mu_{k}}{n+1}\right) .
\end{aligned}
$$

The proof is simply a matter of substituting (7) into the right side of (8) and interchanging the order of summation. The sum easily reduces to $a_{0,0}, \ldots, 0$ which equals the left side of (8).

Suppose $f\left(\theta_{1}, \cdots, \theta_{k}\right) \geqq 0$ and the value of the integral in (8) is unity. Then, dropping all terms in the sum except the one for which $\nu_{1}=\cdots=\nu_{k}=0$,

$$
1 \geqq \frac{1}{(n+1)^{k}} f(0, \cdots, 0) .
$$

Thus

$$
f(0, \cdots, 0) \leqq(n+1)^{k}
$$

for all non-negative functions (7) with average value unity. To estab- 
lish (6), it now suffices to exhibit a function for which equality holds in (9). Let

$$
K_{n}(\theta)=\sum_{n=-n}^{n}\left(1-\frac{|\nu|}{n+1}\right) e^{i \nu \theta}=\frac{1}{n+1}\left(\frac{\sin (n+1) \frac{\theta}{2}}{\sin \frac{\theta}{2}}\right)^{2} .
$$

Then $K_{n}(0)=n+1$ and the desired function is

$$
\begin{gathered}
f\left(\theta_{1}, \cdots, \theta_{k}\right)=K_{n}\left(\theta_{1}\right) \cdots K_{n}\left(\theta_{k}\right) . \\
\text { REFERENCES }
\end{gathered}
$$

1. L. Brickman, A convex class of polynomials and an associated locus problem, J. Math. Anal. Appl. (to appear).

2. L. Fejér, Sur les polynomes trigonométriques, C. R. Acad. Sci. Paris 157 (1913), 571-574.

3. L. Pontrjagin, Topological groups, Princeton Univ. Press, Princeton, N. J., 1946.

4. J. J. Price, Orthonormal sets with non-negative Dirichlet kernels. II, Trans. Amer. Math. Soc. 100 (1961), 153-161.

5. I. J. Schoenberg and G. Szegö, An extremum problem for polynomials, Compositio Math. 14 (1960), 260-268.

6. G. Szegö, Orthogonal polynomials, Amer. Math. Soc. Colloq. Publ. Vol. 23, Amer. Math. Soc., Providence, R. I., 1959.

Cornell Universtty 